\title{
Exercice d'économie du Conseil fédéral et soins ambulatoires
}

\section{Patrick Müller}

Chef de la division Médecine et tarifs ambulatoires

La procédure de consultation sur la deuxième intervention du Conseil fédéral dans le TARMED est terminée. L'Office fédéral de la santé publique décidera dans les prochaines semaines la forme définitive de l'ordonnance. Une chose est certaine: si les mesures entrent définitivement en vigueur au $1^{\text {er }}$ janvier 2018 sans aucun changement, l'offre ambulatoire s'en trouvera grandement affaiblie. Les perdants seront alors en fin de compte les patientes et les patients!

La phase de consultation d'environ trois mois sur la deuxième intervention tarifaire du Conseil fédéral, beaucoup plus importante que la première, s'est terminée le 21 juin 2017. De nombreuses organisations ont profité de cette possibilité pour transmettre leur prise de position à l'Office fédéral de la santé publique (OFSP). Dans une première phase de la consultation, le département Médecine et tarifs ambulatoires de la FMH a mis l'accent sur l'analyse des mesures de cette deuxième intervention et sur la simulation de leurs effets. Les analyses effectuées permettent à la FMH d'évaluer en détail les effets des différentes mesures pour chaque discipline au niveau des positions tarifaires. Ces analyses et simulations étaient nécessaires pour estimer les effets et donc les conséquences sur l'offre ambulatoire en Suisse.

\section{Evaluation générale}

Avec son projet d'ordonnance, le Conseil fédéral n'a pas fait un usage correct de sa compétence subsidiaire, car son intervention dans la structure tarifaire du TARMED n'est pas compatible avec les prescriptions de la LAMal et est donc de nature purement politique. La compétence subsidiaire du Conseil fédéral sert en effet à corriger une adéquation manquante, mais ne devrait pas déformer ou péjorer encore plus la structure tarifaire. Or l'intervention tarifaire vise uniquement à réduire unilatéralement les coûts, ce qui n'a rien à voir avec le fait de restaurer l'adéquation. Lors de sa première intervention en 2014, le Conseil fédéral avait déjà enfreint l'obligation légale d'adéquation, comme l'a confirmé le 29 mai 2017 un arrêt du Tribunal arbitral du canton de Lucerne ${ }^{1}$, donnant raison à la partie plaignante. Dans son arrêt, le Tribunal a également souligné que toute intervention tarifaire du Conseil fédéral devait également être appropriée conformément à la LAMal.

Dans le domaine opératoire notamment, l'opérateur est parfois confronté à de longs moments d'attente durant lesquels il ne peut pas fournir d'autres prestations.

Beaucoup de mesures ont notamment pour effet un abaissement disproportionné de la prestation médicale (PM) par rapport à la prestation technique (PT). Pourtant, la spécialisation du personnel non médical a abouti ces dernières années à une forte augmentation des coûts dans les cabinets médicaux et les hôpitaux; et comme aucune compensation par une adaptation

\section{Faites une simulation des effets} sur votre liste de prestations!

Que signifient I'intervention tarifaire du Conseil fédéral et les mesures proposées pour mon propre cabinet? Dois-je m'attendre à des pertes? Le département Médecine et tarifs ambulatoires reçoit actuellement chaque jour de telles questions.

L'outil Volumis Online soutient tous les membres de la FMH dans la recherche d'une réponse à ces questions et permet à chaque médecin de simuler les effets de l'intervention tarifaire sur leur catalogue de prestations individuel.

Vous trouverez d'autres informations sur myFMH et sur notre site internet: www.fmh.ch $\rightarrow$ Tarifs ambulatoires $\rightarrow$ TARMEDTarif $\rightarrow$ Modifications TARMED dès le 1.1.2018. 
des tarifs dans le domaine de la PT n'a eu lieu depuis 2004, cette hausse des coûts est déjà aujourd'hui subventionnée transversalement par la «prestation médicale». En outre, l'intervention ne prévoit aucune mesure permettant de corriger les prestations sous-tarifées et inappropriées et de revaloriser la prestation TARMED, par exemple la suppression de la division par deux du taux de coûts des 5 dernières minutes (position 00.0030) ou l'introduction d'une indemnité forfaitaire de dérangement durant la consultation.

\section{Pour la FMH, l'application des limitations de facturation est extrêmement problématique.}

Dans leur ensemble, les mesures proposées mènent à un net affaiblissement de la médecine ambulatoire, ne donnent aucune incitation à fournir des prestations dans le domaine ambulatoire, nettement moins cher et plus efficace, et mettent en péril la prise en charge ambulatoire de la population des régions périphériques, hors des centres urbains, prise en charge qui est déjà problématique aujourd'hui. Ainsi, le Conseil fédéral va à l'encontre du principe politique adopté, «l'ambulatoire avant l'hospitalier».

\section{Remarques concernant diverses mesures de l'intervention tarifaire}

La FMH commente ci-après les trois mesures de l'ordonnance qui ont les plus grandes répercussions du point de vue du corps médical. La prise de position détaillée de la FMH remise au Conseil fédéral et à l'OFSP est publiée sur le site internet de la FMH: www.fmh.ch $\rightarrow$ Tarifs ambulatoires $\rightarrow$ TARMED Tarif $\rightarrow$ Modifications TARMED dès le 1.1.2018.

\section{Abaissement de la "valeur intrinsèque quantitative»}

La "valeur intrinsèque quantitative» permet une compensation de la durée de formation postgraduée et continue (notamment celle liée à une réduction de la durée de la vie professionnelle) par spécialité. Pour la FMH, il est incontesté que les salaires des médecinsassistants et chefs de clinique ont augmenté depuis l'introduction du TARMED et que ces médecins sont depuis 2005 assujettis à la loi sur le travail. Néanmoins, ce qui est encore déterminant aujourd'hui pour le revenu perçu sur l'ensemble de la vie, c'est le temps que le médecin doit investir dans sa formation postgraduée et continue: si pour un titre de «médecin praticien" il faut trois ans de formation postgraduée, pour un titre de spécialiste, il faut au moins 5 ou 6 ans.
L'abaissement général pour toutes les disciplines avec un facteur de valeur intrinsèque en dessous de 1.0 n'est pas compréhensible pour la FMH, car il est en contradiction avec le principe de l'adéquation et ne signifie rien d'autre qu'une véritable réduction de la rémunération de $3,2 \%$.

Du point de vue de la FMH et des associations faîtières affiliées, il convient de continuer à recourir à des différenciations de "valeur intrinsèque quantitative», car de nombreuses prestations requièrent une formation postgraduée supplémentaire voire un deuxième titre de spécialiste. Si l'on souhaite maintenir la fourniture de prestations spécialisées qui demandent beaucoup d'expérience, on doit alors compenser les différences de «revenu durant la vie active» dues à des périodes de formation postgraduée plus longues. A ce sujet, la FMH a élaboré, avec les associations faîtières affiliées, un concept approprié et économique, qui sera mis en œuvre dans le cadre du projet TARCO.

\section{Augmentation de la "productivité médicale» dans les unités opératoires}

La "productivité médicale» doit compenser le temps pour lequel un médecin ne peut pas appliquer directement un tarif dans le cadre de ses activités relevant du TARMED, autrement dit, où la facturation de positions du TARMED n'est pas possible. Dans le domaine opératoire notamment, l'opérateur est parfois confronté à de longs moments d'attente durant lesquels il ne peut pas fournir d'autres prestations. En outre, la productivité médicale prend en compte aujourd'hui les prestations qui ne peuvent pas être directement facturées au patient, par ex. le «sign-in», le «sign-out», les temps de changement, les réunions d'équipe ou les rapports et les préparatifs d'opération en présence de l'opérateur (anesthésie et positionnement en présence de l'opérateur). La productivité en salle d'opération baisse en conséquence.

Une hausse de la productivité médicale en salle d'opération, comme le Conseil fédéral la prévoit, ne peut être obtenue qu'en relation avec la tarification des mesures préopératoires et du suivi postopératoire. C'est ce qui est prévu dans le projet TARCO. Mais avant que ces facteurs, dont le tarif ne tient pas compte aujourd'hui, puissent être pris en considération, la "productivité médicale» pour le domaine opératoire doit de nouveau être abaissée à son niveau originel. Sans prise en compte, dans la structure tarifaire, des prestations fournies en plus par l'opérateur, qui étaient jusqu'ici indemnisées par la productivité inférieure, une augmentation ne serait ni appropriée ni économique. 


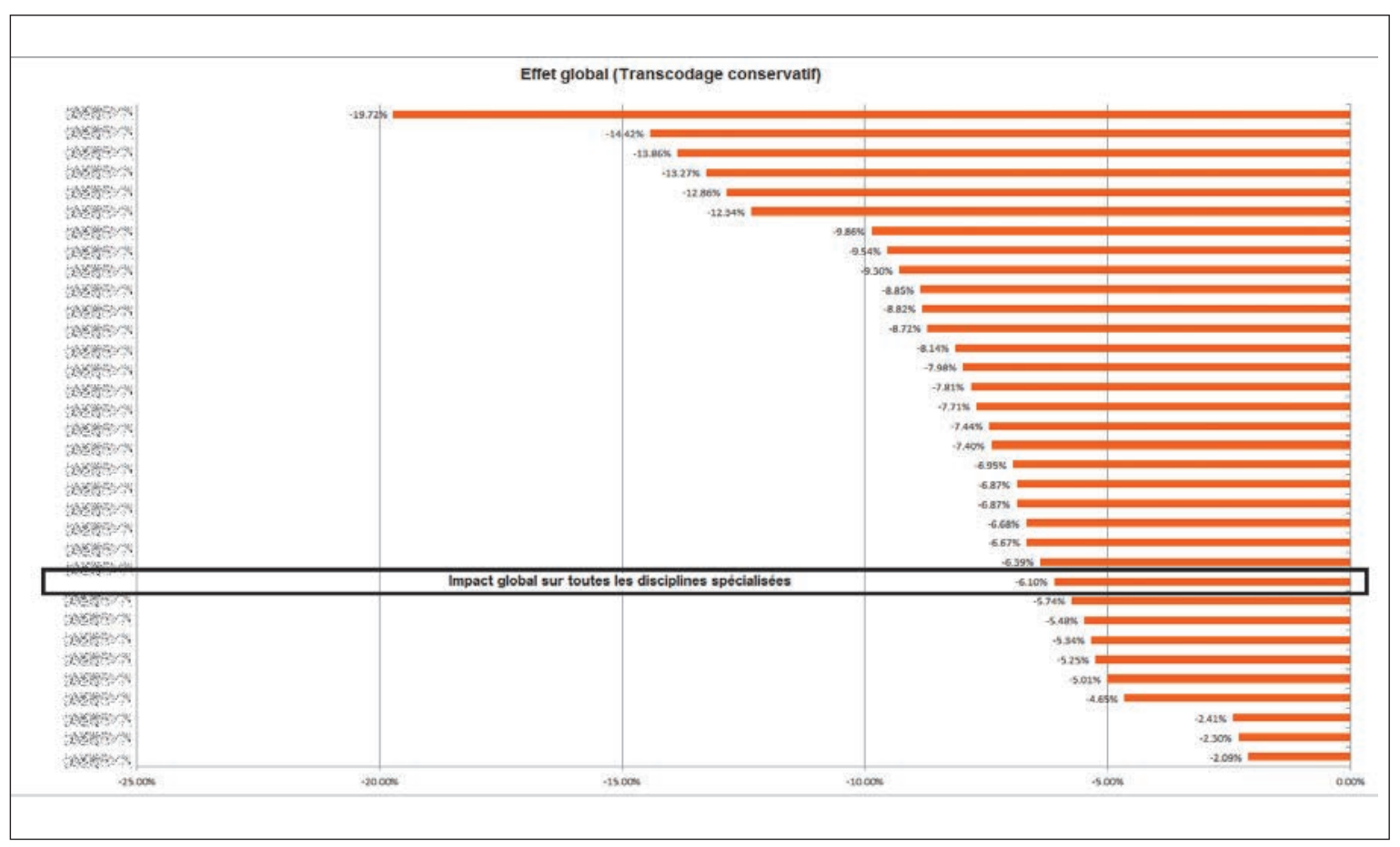

Exercice d'économie du Conseil fédéral: impact global de la deuxième intervention tarifaire pour chaque discipline (total des points tarifaires).

\section{Application des limitations}

Pour la FMH, l'application des limitations de facturation est extrêmement problématique. Avec l'introduction de nouvelles limitations, en particulier sur les prestations médicales de base ainsi que sur la consultation et la prestation médicale en l'absence du patient, on introduit un rationnement caché. Il faut savoir en effet que, en particulier chez les enfants et les adolescents, chez les personnes âgées ou celles atteintes de troubles psychiques, les entretiens en l'absence du patient avec les proches, d'autres professionnels de la santé et les autorités constituent une partie importante et indispensable du traitement médical. Ces limitations globales créent un faux incitatif. Et ce sont finalement les patients qui sont les perdants, car le travail interprofessionnel et interdisciplinaire pour le patient est ainsi rendu plus difficile.

\section{Chaque intervention tarifaire modifie les incitatifs.}

La FMH demande expressément au Conseil fédéral de vérifier et de repenser les limitations dans chaque cas particulier, avec les experts concernés des sociétés de discipline et la FMH.

La FMH a analysé les effets de chaque mesure et l'impact global de la deuxième intervention tarifaire sur chaque discipline et a procédé à une simulation de ces effets à l'aide d'un transcodage élaboré par ses soins. Sur le graphique ci-après, on peut voir ces effets en fonction des différentes disciplines. Une barre y correspond à une discipline. Pour des raisons de protection des données, les noms des disciplines ont été rendus illisibles. Une chose est certaine: elles sont toutes touchées par l'intervention tarifaire!

\section{Stabiliser les primes AOS par un financement uniforme}

Chaque intervention tarifaire modifie les incitatifs. Dans le cas présent, il faut s'attendre à un transfert, vers le domaine hospitalier, des prestations qui ne peuvent plus être fournies à prix coûtant dans le domaine ambulatoire. Est-ce éventuellement ce que souhaite le Conseil fédéral, afin d'obtenir un soulagement apparent des primes AOS? Au cours des vingt dernières années, les primes AOS ont augmenté de près de $50 \%$ de plus que les coûts de la santé ${ }^{2}$. Cette évolution inégale est due aux différents modèles de financement. Alors que le financement des soins hospitaliers est dual-fixe (par les cantons et les assureurs), celui des soins ambulatoires est moniste (assuré uniquement par les assureurs, à la charge des primes AOS). L'intervention tarifaire menace le transfert visé et expressément voulu du domaine hospitalier vers le domaine ambulatoire, meilleur marché et plus judicieux 


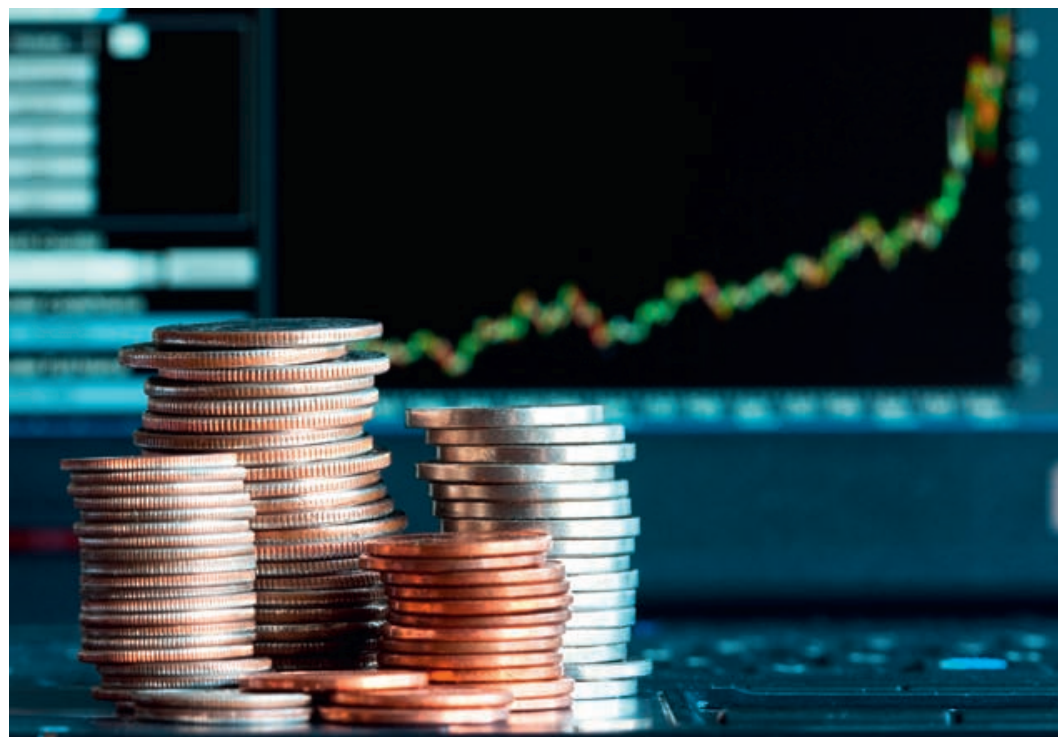

Au cours des vingt dernières années, les primes AOS ont augmenté de près de $50 \%$ de plus que les coûts de la santé.

Correspondance: FMH / Division Médecine et tarifs ambulatoires Baslerstrasse 47

$\mathrm{CH}-4600$ Olten

Tél. 0313591230 Fax 0313591238

tarife.ambulant[at]fmh.ch sur le plan économique, et elle contredit donc la stratégie Santé2020 du Conseil fédéral. Voulant réduire la hausse annuelle des primes, les politiques s'attaquent une fois de plus aux symptômes au lieu d'éliminer les faux incitatifs du système.

\section{Restaurer l'adéquation n'est possible que par une révision globale}

Cette deuxième intervention tarifaire du Conseil fédéral, très étendue, n'augmente pas la disposition des associations d'assureurs-maladie à collaborer activement et avec engagement à une révision globale. Car l'exercice d'économie du Conseil fédéral promet un allègement des primes de 700 millions de francs, ce qui diminue le volume de comparaison AOS pour les né- gociations sur la révision globale. Pour la FMH et les organisations représentées à la Chambre médicale, la nécessité de réviser la structure tarifaire ambulatoire TARMED introduite en 2004 est incontestée. Après le rejet de la première proposition de révision lors de la votation générale des membres en mai 2016, la FMH a lancé le projet de révision TARCO en vue d'apporter des améliorations. Le but poursuivi par TARCO est une tarification appropriée et économique de toutes les prestations médicales ambulatoires. Car, du point de vue du corps médical, une révision tarifaire globale avec toutes les organisations de médecins et les partenaires tarifaires est la seule voie correcte. La FMH poursuit le projet TARCO avec beaucoup d'engagement.

\section{Perspectives}

Ces prochaines semaines, le Conseil fédéral annoncera l'ordonnance et les mesures définitives; leur entrée en vigueur est prévue pour le $1^{\text {er }}$ janvier 2018. Vu les importantes modifications, qui toucheront chaque médecin, il sera non seulement nécessaire de changer ou d'adapter les logiciels de saisie des prestations et de facturation, mais il sera aussi indispensable d'effectuer une vérification des durées de consultation et de traitement à la suite des nouvelles limitations. Les organisations de médecins affiliées à la FMH et les membres individuels de la FMH seront préparés par des informations ciblées, une liste de questions fréquentes (FAQ), des fiches d'information, un navigateur tarifaire et des publications à ce sujet. La FMH informera à nouveau après l'annonce de l'ordonnance définitive.

\section{Crédits illustration}

Graphique: Transcodage FMH, basé sur les données de NewIndex avril 2017

Photo: @ Kenishirotie | Dreamstime.com 